\title{
Maria Dell'Isola, L'ultima profezia. La crisi montanista nel cristianesimo antico
}

Trapani, Il Pozzo di Giacobbe (Oi christianoi. Sezione antica 30), 2020, pp. 182, ISBN 978-88-6124-802-1, € 22.

\section{Gaetano Spampinato}

\section{(2) OpenEdition}

\section{Journals}

\section{Edizione digitale}

URL: https://journals.openedition.org/mythos/3789

DOI: $10.4000 /$ mythos.3789

ISSN: 2037-7746

Editore

Salvatore Sciascia Editore

\section{Notizia bibliografica digitale}

Gaetano Spampinato, «Maria Dell'Isola, L'ultima profezia. La crisi montanista nel cristianesimo antico», Mythos [Online], 15 | 2021, online dal 15 décembre 2021, consultato il 15 décembre 2021. URL: http:// journals.openedition.org/mythos/3789 ; DOI: https://doi.org/10.4000/mythos.3789

Questo documento è stato generato automaticamente il 15 décembre 2021.

Mythos 


\section{Maria Dell'Isola, L'ultima profezia. La crisi montanista nel cristianesimo antico}

Trapani, Il Pozzo di Giacobbe (Oi christianoi. Sezione antica 30), 2020, pp. 182, ISBN 978-88-6124-802-1, € 22.

\section{Gaetano Spampinato}

\section{NOTIZIA}

Maria Dell'Isola, L'ultima profezia. La crisi montanista nel cristianesimo antico Il Pozzo di Giacobbe (Oi christianoi. Sezione antica 30), Trapani, 2020, 182 pp., ISBN 978-88-6124-802-1, $22 €$

1 Il titolo della monografia di M. Dell'Isola, L'ultima profezia. La crisi montanista nel cristianesimo antico, situa il contributo, come la studiosa stessa riconosce, "sulla scia di tutti i lavori che lo hanno preceduto" (p. 7), proponendo un'analisi del fenomeno montanista in prospettiva storica, letteraria e religiosa. Tuttavia, a differenza degli studi precedenti, questo volume si presenta come un esame più specifico e, per certi versi, originale. Mentre le monografie precedenti si concentrano in generale sul montanismo, sulle sue caratteristiche più rilevanti o sull'opposizione della Grande Chiesa $^{1}$, il libro di Dell'Isola (frutto di una tesi di dottorato presso la Fondazione San Carlo di Modena e il Max-Weber-Kolleg di Erfurt) ruota attorno a due nuclei principali: da un lato, l'analisi degli oracoli montanisti, del loro contenuto, del lessico utilizzato e dei rimandi alle Scritture (capitoli I e II); dall'altro, lo studio della costruzione del montanismo in quanto eresia da parte degli eresiologi, in particolare Epifanio di Salamina (capitolo III). Queste due parti, che a un primo sguardo possono sembrare due studi autonomi, nell'argomentazione dell'autrice sono strettamente connesse e l'una è necessaria per comprendere l'altra: infatti, la costruzione polemica dell'eresiologia è spesso il quadro in cui leggere e interpretare gli oracoli pervenuti. 
2 Nell'introduzione, la studiosa ricostruisce per sommi capi il contesto storico e religioso del movimento, gli oracoli del montanismo, la loro dimensione intertestuale e il rapporto tra eresiologia e montanismo. In questa rassegna, si mettono in evidenza i problemi e i limiti degli studi precedenti. Ad esempio, riguardo alla definizione del montanismo come eresia, Dell'Isola ricorda il contributo di M. Pesce sulla problematicità dell'utilizzo del termine "eresia" nello studio dei diversi movimenti nel cristianesimo antico, termine che appare fortemente connotato in senso teologico e dottrinale, mettendo in guardia rispetto a una costruzione storica fondata sulle fonti eresiologiche ${ }^{2}$. Tuttavia, la studiosa ricorda che "il punto di vista dell'eresiologo è comunque imprescindibile per qualsiasi tentativo di comprensione dell'eretico, perché offre comunque uno spaccato attendibile sulla percezione che si aveva sull'avversario" (pp. 23-24). In altre parole, Dell'Isola dichiara che nella sua analisi la prospettiva eresiologica è centrale poiché fornisce una importante chiave di lettura, anche se polemica, sul montanismo.

3 Un altro tema importante dell'introduzione, ripreso ampiamente nel secondo capitolo, riguarda l'intertestualità negli oracoli montanisti. La studiosa rimanda a un contributo di D.E. Groh dedicato alla questione del "rapporto tra oracoli montanisti e Scrittura partendo dall'ipotesi dell'esegesi carismatica che caratterizzerebbe sia gli oracoli sia il

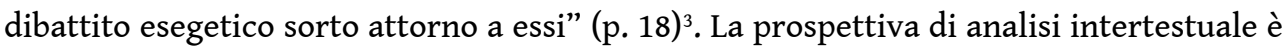
di particolare interesse, pur con i limiti che la studiosa stessa metterà in luce, perché permette di esplorare un panorama più ampio di scritti e ricostruire la possibile interazione tra di essi. Tale prospettiva si rileva utile anche sul piano dell'indagine attorno alle "eresie" e alle catalogazioni proposte dagli eresiologi perché "sottolinea come, abbandonando le operazioni retoriche della letteratura eresiologica [...] sia possibile mettere in comunicazione testi, e quindi gruppi, che la rigida classificazione eresiologica tende a considerare distanti e inconciliabili" (p. 22).

4 Come detto, i primi due capitoli sono dedicati all'analisi e al commento degli oracoli montanisti e al raffronto con alcuni passi scritturistici. Il primo capitolo, "Gli oracoli dei montanisti: contenuti e contesti”, dedicato allo studio di tutti gli oracoli dei montanisti a noi pervenuti rappresenta, a mio avviso, una delle sezioni più originali del libro. La stessa autrice, all'inizio del capitolo, nota come l'analisi degli oracoli avesse rappresentato per tanto tempo una "lacuna considerevole nella ricerca sulla storia del montanismo" (p. 31): gli oracoli sono stati spesso analizzati in opere generali dedicate al montanismo o al profetismo antico (e.g., quella di D.E. Aune) ${ }^{4}$, oppure tradotti senza un'analisi più approfondita - per esempio, nell'utilissima raccolta di R.E. Heine ${ }^{5}$. Dell'Isola ricorda il lavoro di H.E. Mader, che aveva proposto un esame degli oracoli montanisti incentrato, però, soltanto su alcuni degli oracoli (quelli tramandati da Epifanio in Panarion 48 e da Eusebio in Storia ecclesiastica V 16, 17), nel tentativo di ricostruire una immagine fedele del movimento originario ${ }^{6}$. L'analisi di Mader si scontra con la stessa questione con cui si erano scontrati altri studiosi prima di lei: il problema del criterio dell'autenticità degli oracoli montanisti. L'attenzione nei confronti di chi ha pronunciato l'oracolo e dell'affidabilità delle fonti - criterio già criticato da W. Tabbernee ${ }^{7}$ - ha spesso ostacolato una lettura complessiva della produzione oracolare catafrigia. Dell'Isola, contro questo criterio di cui mette bene in evidenza i limiti, dichiara di volere analizzare tutti gli oracoli tramandati dalle fonti letterarie, sebbene si mostri consapevole della problematica attribuzione di alcuni di essi - ad esempio, quello citato da Celso (cf. Origene, Contro Celso VII 9). In questo modo, 
la studiosa fornisce una visione globale del profetismo montanista, in senso cronologico, con autori che vanno dalla fine del II secolo fino al IV-V secolo, e geografico, includendo le testimonianze di Tertulliano, spesso escluse dalle trattazioni precedenti.

5 L'analisi degli oracoli montanisti è molto approfondita: per ogni testo, Dell'Isola propone una traduzione e un commento delle singole parole o espressioni adoperate, cogliendo le diverse sfumature lessicali. Grande attenzione viene data anche - punto di forza di quest'analisi - allo studio della fonte in cui vengono citati i diversi oracoli. Mentre infatti una ricostruzione del contesto degli oracoli in sé risulta piuttosto difficile, a causa innanzitutto della loro natura frammentaria, i riferimenti all'autore che cita l'oracolo e ai precisi passi delle opere dove essi vengono menzionati consentono di guardare a questi ultimi nella prospettiva funzionale con cui sono utilizzati. Se, per esempio, Tertulliano in Esortazione alla castità 10,5 menziona un oracolo di Prisca per spiegare gli effetti della purificantia, Epifanio, nel contesto eresiologico del Panarion $(48,4,1)$, cita un oracolo di Montano per sottolinearne la follia e la distanza rispetto alla vera profezia. In questa operazione polemica, come nota la studiosa, spesso l'eresiologo modifica il senso di un oracolo per dare più efficacia alle proprie accuse.

6 A questo proposito, possiamo proporre uno spunto per ulteriori riflessioni. Come detto, l'analisi di Dell'Isola guarda al lessico e ai riferimenti interni dei vari oracoli e riesce a osservare concretamente gli aspetti più importanti del montanismo. In alcuni casi, Dell'Isola ricorda anche alcuni paralleli con la coeva produzione cristiana antica. Un raffronto con la produzione letteraria e con l'ambito oracolare della religiosità tradizionale greca e latina potrebbe approfondire ulteriori aspetti di quest'analisi, anche sul piano della costruzione eresiologica. La questione delle "origini religiose" del montanismo ha a lungo coinvolto gli studiosi; sebbene tale prospettiva appaia oggi superata in nome di una visione più dinamica, proprio sul piano eresiologico essa potrebbe fornire alcuni spunti interessanti. Un esempio di questo discorso viene dal menzionato oracolo citato da Epifanio, dove l'uomo è descritto come una lira suonata Dio. La metafora del profeta come strumento musicale, come nota la studiosa, è presente anche in altri autori cristiani; qui, però, Epifanio la sottolinea per criticare la maniera profetica montanista, in cui il profeta cade in un'estasi incosciente e diventa strumento inerme del dio. Tale motivo polemico riguarda anche la profezia pagana, spesso accusato da parte dei cristiani di avere origine demoniaca e di realizzarsi in forme estatiche esagerate, in cui il dio si impossessa del proprio strumento. Il contesto microasiatico, più nello specifico frigio, di origine e diffusione del montanismo potrebbe portare ad alcuni raffronti, anche e soprattutto sul piano della costruzione polemica, attorno agli oracoli montanisti.

7 Nel secondo capitolo del libro ("La presenza di testi scritturistici negli oracoli montanisti: una discussione sui legami testuali”), Dell'Isola analizza gli eventuali legami tra gli oracoli montanisti e alcuni passi scritturistici, distinguendo "tra citazioni (rimando esplicito), allusioni (rimando implicito) ed echi (affioramento involontario)" (p. 92). Anche in questo caso l'analisi è precisa e puntuale, e guarda alle immagini, alle espressioni e ai termini utilizzati negli oracoli e nei passi scritturistici di riferimento. La studiosa si concentra principalmente sulla Scrittura canonica, ovvero quegli "scritti che entreranno a fare parte del canone soltanto in un'epoca successiva a quella che vede la nascita e lo sviluppo del montanismo primitivo, nonostante alcuni godessero già allora 
di un implicito grado di autorità" (p. 27). Anche in questo caso, un raffronto con la più larga produzione scritturistica del periodo e con altri testi non entrati nel canone potrebbe ulteriormente arricchire l'analisi: del resto, nell'introduzione Dell'Isola aveva ricordato il contributo di N. Denzey Lewis e la sua ipotesi di un retroterra religioso comune tra gli oracoli montanisti e alcuni testi di Nag Hammadi ${ }^{8}$. Ad ogni modo, l'analisi di Dell'Isola dimostra come, sebbene esistano immagini e termini comuni ad alcuni passi scritturistici e agli oracoli montanisti, i contesti degli uni e degli altri si rivelino il più delle volte diversi, il che rende difficile parlare di veri e propri riferimenti o citazioni; invece, come afferma la studiosa nelle conclusioni, "la diversità del contesto [...] ha dunque messo in luce la necessità di ridefinire i contorni del fenomeno di ripresa testuale, che più che intenzionale appare frutto invece di un affioramento involontario e spontaneo" (p. 154).

L'ultimo capitolo del libro ("Il montanismo nella letteratura eresiologica: i contorni di una rappresentazione") è incentrato sulla costruzione del montanismo nelle fonti eresiologiche. Questo capitolo è importante per meglio comprendere gli altri due, perché affronta quel contesto eresiologico dove sono menzionati gli oracoli. Tramite un'analisi discorsiva delle fonti, Dell'Isola dimostra quali siano i punti più importanti nella descrizione polemica dei catafrigi, sottolineando sul piano semantico e lessicale alcune coppie oppositive attorno alle quali prendono forma il discorso eresiologico e il confronto con i veri profeti: 1. senno e intelligenza / mancanza di senno e intelligenza; 2. significato conoscibile / inconoscibile; 3. chiarezza / oscurità; 4. (verità) / falsità. I risultati di questo attento esame vengono estesi ad altri casi polemici: la studiosa, infatti, dedica un interessante paragrafo al confronto con la descrizione polemica di Marco il Mago da parte di Ireneo di Lione. In questo caso, Dell'Isola nota come, oltre ai punti descritti, le rappresentazioni di Montano da un lato e Marco dall'altro presentino altri aspetti polemici comuni: l'inganno operato dai due eretici; la seduzione nei confronti delle donne; il loro parlare ingannatore che li avvicina ai pagani.

A tal proposito, la studiosa, citando A. Le Boulluec ${ }^{9}$, ricorda come tali strategie fossero comuni nelle polemiche religiose dell'epoca: esse, per esempio, vengono utilizzate dai cristiani nella rappresentazione dei pagani, ma anche questi ultimi spesso le adoperano. L'autrice si limita a menzionare la polemica contro alcuni presunti profeti da parte di Celso, ma potremmo anche ricordare l'ironia di Luciano contro il falso profeta Alessandro di Abonouteichos o l'indignazione di Plutarco nei confronti di alcuni profeti imbroglioni soliti attirare donne e fanciulli fuori dai santuari di Serapide e Cibele (Sull'oracolo della Pizia 407C-D). Questi esempi mostrano come le accuse contro i profeti impostori presentassero alcuni aspetti comuni alla maggior parte delle descrizioni polemiche di presunte figure carismatiche. A tal proposito, nell'ultimo paragrafo del capitolo la studiosa segnala le trasformazioni della rappresentazione eresiologica dei catafrigi fino alla Tarda Antichità. Alle tradizionali accuse antimontaniste se ne aggiunsero altre generiche contro i gruppi eretici, come l'ingordigia o la lussuria. Così si arriva alla descrizione paradossale di Timoteo di Costantinopoli, che "pur riproponendo la tesi tradizionale dell'annullamento dei matrimoni [scil. per l'eccessivo rigore morale dei catafrigi], allo stesso tempo definisce Prisca e Massimilla come due pornikái» (p. 148).

Per concludere, l'analisi proposta dalla studiosa è di grande interesse per comprendere la costruzione polemica del fenomeno montanista da parte degli eresiologi e come un movimento nato in Frigia verso la metà del II secolo venne percepito, tramite 
cambiamenti e riadattamenti in chiave polemica, da autori molto distanti. È proprio questa prospettiva che costituisce uno dei tratti più originali del libro di Dell'Isola, che fornisce un nuovo studio, chiaro e ben scritto, su un fenomeno che continua a rivelarsi sempre stimolante. Si potrebbe, per concludere, capovolgere il giudizio di A. Faggiotto, ricordato nella premessa del volume, per il quale sull'eresia dei frigi era stato scritto fin troppo ${ }^{10}$ : speriamo che sul montanismo si continui a scrivere ancora.

\section{NOTE}

1. Per esempio, C. TReVeTt, Montanism: Gender, Authority and the New Prophecy, Cambridge 1996 e W. TABBERNEE, Fake Prophecy and Polluted Sacraments: Ecclesiastical and Imperial Reactions to Montanism, Leiden-Boston 2007.

2. M. PESCE, «La relazione tra il concetto di eresia e la storia del cristianesimo», Annali di Storia dell'Esegesi 31/1 (2014), 151-168.

3. D.E. GROH, «Utterance and Exegesis: Biblical Interpretation in the Montanist Crisis», in D.E. Groh - R. Jewett (eds.), The Living Text. Essays in Honor of Ernest W. Saunders, Lanham 1985, 73-95.

4. D.E. AUNE, Prophecy in Early Christianity and the Ancient Mediterranean World, Grand Rapids 1983.

5. R.E. HEINE, The Montanist Oracles and Testimonia, Macon 1989.

6. H.E. M ADER, Montanistische Orakel und kirchliche Opposition: Der frühe Streit zwischen den phrygischen "neuen Propheten" und dem Autor der vorepiphanischen Quelle als biblische Wirkungsgeschichte des 2. Jh. n.Chr, Göttingen 2012.

7. W. T ABBERNEE, "“Recognizing the Spirit": Second-generation Montanist Oracles», Studia Patristica 40 (2006), 521-526.

8. N. Denzey LeWIS, «What Did the Montanists Read?», Harvard Theological Review 94 (2001), 427-448.

9. A. Le BOULlueC, La notion d'hérésie dans la littérature grecque. II ${ }^{e}-I I^{e}$ siècles, Paris 1985, 115.

10. A. FAGGiotTo, L'eresia dei frigi, Roma 1924, 3.

\section{AUTORI}

\section{GAETANO SPAMPINATO}

Institut du Monde Antique et Byzantin

Rue Pierre-Aeby 16, 1700 Fribourg, Switzerland

NFS Project "Religious Competition in Late Antiquity"

gaetano.spampinato(at)unifr.ch 